\title{
Beratung im Aktivierungsparadigma
}

\section{Macht die Eingliederungsvereinbarung den Unterschied?}

\section{Carolin Freier}

Eingegangen: 4. Mai 2020 / Angenommen: 7. Dezember 2020 / Online publiziert: 21. Mai 2021

(C) Der/die Autor(en) 2021

Zusammenfassung Beratung im Jobcenter unterscheidet sich von anderen Beratungssituationen u. a. durch den Abschluss einer Eingliederungsvereinbarung. Dies sind rechtsverbindliche Verträge zwischen Jobcenter und Arbeitsuchenden, die das Prinzip „Fördern und Fordern“ in der aktivierenden Sozialpolitik umsetzen sollen. Eingliederungsvereinbarungen werden insbesondere kritisch diskutiert, da in ihnen die Elemente des Forderns betont und gleichzeitig die Arbeitsinteressen und Lebensbedürfnisse der Arbeitssuchenden nur partiell eingebracht werden. $\mathrm{Ob}$ die Eingliederungsvereinbarung eine zentrale Rolle einnimmt, um das Fordern in der Beratungssituation zu verankern, untersucht der Beitrag unter den Bedingungen eines Feldexperimentes. Darin wurden der Einsatz der Eingliederungsvereinbarung und die Möglichkeit, auf ihrer Basis zu sanktionieren, zufällig variiert. Interaktionsbeobachtungen und semistrukturierte Interviews mit Beratungspersonal und Arbeitsuchenden werden im Anschluss an die Rahmenanalyse sowie den dramaturgischen Ansatz von Erving Goffman analysiert. Der Beitrag zeigt: Ob das Beratungsgespräch eher fordernd oder unterstützend angelegt ist, wird weniger durch den Abschluss oder Nichtabschluss der Eingliederungsvereinbarung beeinflusst, sondern vielmehr durch die Rahmung des Beratungsgesprächs und das ,,impression management“ Arbeitsuchender.

Schlüsselwörter Interaktionsanalyse · Impression Management · Hilfe und Kontrolle $\cdot$ Sozialpolitik $\cdot$ Jobcenter 


\title{
Counselling in the activation paradigm
}

Does the back-to-work agreement make the difference?

\begin{abstract}
Counselling at the job centre typically ends with the signing of backto-work agreements. These are legally binding contracts between jobcentres and jobseekers, which are intended to implement the "carrot and stick" elements of activation policy. Back-to-work agreements have been discussed critically because they emphasize elements of control and because jobseekers can only partially contribute their work interests and life needs. This article examines whether the back-to-work agreement plays a central role in establishing control in the counselling situation under the conditions of a field experiment. Within this field experiment, the use of backto-work agreements and the possibility of sanctioning on its basis were randomly varied. Interaction observations and semistructured interviews with counselling staff and jobseekers are analysed following the framework analysis and the dramaturgical approach of Erving Goffman. The article shows that whether the counselling is more controlling or supportive is influenced less by the fact of contracting or not contracting the back-to-work agreement. The crucial factor instead is the framing of the counselling interview and the impression management of jobseekers.
\end{abstract}

Keywords Interaction Analysis · Impression Management - Social Support and Control · Social Policy · Public Employment Services

\section{Beratung von Arbeitsuchenden in Jobcentern}

Um Arbeitsuchende ${ }^{1}$ zu ermutigen und zu befähigen, statt sie in ihren Möglichkeiten und den Schwierigkeiten der Lebensbewältigung zu übergehen, braucht es ,,die Vermittlung der Arbeitsinteressen mit der Anerkennung von Lebensbedürfnissen und -kompetenzen, die darüber hinausgehen“ (Thiersch 2013, S. 214). An diesem Anspruch Sozialer Arbeit in der Jugendberufshilfe sollte sich auch die Beratung in der Arbeitsvermittlung messen lassen. Denn auch hier werden die weiteren Erwerbsbiographien der Beratenen und damit die Lebensqualität entscheidend geprägt. In der Beratungspraxis kommen die Interessen der Arbeitsuchenden jedoch nicht immer zur Sprache (Sondermann 2013; Grimmer 2018).

Im Zusammenhang damit steht die Besonderheit der Beratung in der Arbeitsvermittlung der Grundsicherungsträger (Sozialgesetzbuch II/SGB II). Diese unterliegt den politischen Leitplanken des Aktivierungsparadigmas: Die aktivierende Sozialpolitik geht in Deutschland mit an Bedingungen geknüpften Bezügen von Sozialleistungen (Lessenich und Mau 2005), einer höheren Individualisierung von strukturellen Arbeitsmarktrisiken und der Vermarktlichung der Wohlfahrtsversorgung einher (Nullmeier 2004; Henning 2011; Lessenich 2012). So verbindet das Aktivierungsparadigma - insbesondere im Prinzip „Fördern und Fordern“ - die erhöhte Notwendigkeit erwerbstätig zu sein einerseits mit der Förderung von Beschäftigungsfähigkeit

\footnotetext{
${ }^{1}$ Der Begriff „Arbeitsuchende“ wird im Folgenden für Personen verwendet, die in Jobcentern arbeitslos gemeldet sind und gleichzeitig Arbeitslosengeld II beziehen.
} 
andererseits und stärkt damit die Eigenverantwortung der Arbeitsuchenden (siehe zur kritischen Diskussion insbesondere hinsichtlich der Indienstnahme Sozialer Arbeit: Klammer et al. 2017; Thiersch 2013, S. 215; Dahme et al. 2008, S. 273 f.; Galuske und Rietzke 2005). „Aktivierung“ umschreibt dabei eine Kombination aus Anreizen und Sanktionen, um erwerbslose Menschen in den Arbeitsmarkt zu (re-)integrieren (Etges und Lenger 2010, S. 331).

Vor diesem Hintergrund ist die Beratung in der Arbeitsvermittlung in dreierlei Hinsicht besonders. Erstens ist die Beratung nicht zieloffen, sondern bezweckt, Arbeitsuchende zur Erwerbstätigkeitsaufnahme zu aktivieren. Zweitens ist die Beratung nicht freiwillig, sondern deren Inanspruchnahme und Mitwirkung ist eine der Bedingungen, um Arbeitslosengeld-II-Leistungen der steuerfinanzierten Grundsicherung (SGB II) zu erhalten. Drittens findet die Beratung im Rahmen einer Vertragsbeziehung der Arbeitsuchenden mit dem Jobcenter statt. Der Besprechung der Arbeitsbiografie und des Zielberufes folgt der Abschluss eines öffentlich-rechtlichen Vertrages - der sog. „Eingliederungsvereinbarung“ ( 15 SGB II) -, in der die Handlungsstrategie zur Arbeitsmarktintegration festgehalten wird. Die Eingliederungsvereinbarung (EGV) soll das Prinzip „Fördern und Fordern“ umsetzen, indem in ihr sowohl Leistungen des Jobcenters als auch Pflichten der Arbeitsuchenden fixiert werden. Sie wird einerseits als Chance gesehen, Kooperation und Mitsprachechancen und damit soziale Rechte der Arbeitsuchenden vertraglich festzuhalten (Banafsche 2013; Weinbach 2012; Bieback 2009). Die Forschung zeigt jedoch, dass aufgrund eines asymmetrischen Machtverhältnisses Arbeitsuchende ihre sozialen Rechte und Autonomie kaum realisieren (Betzelt und Bothfeld 2014; Marquardsen 2011; Bieback 2009).

Ob die EGV in diesem asymmetrischen Machtverhältnis eine entscheidende Rolle spielt, um Elemente des Forderns in der Beratung zu verankern, untersucht dieser Beitrag. Der Einfluss des Vertragsdokumentes auf die Beratungspraxis im SGB II kann erstmals im Rahmen eines Feldexperimentes des Instituts für Arbeitsmarktund Berufsforschung untersucht werden (Bernhard et al. 2019). Dabei wurde zufällig variiert, ob und welche EGVs abgeschlossen werden - und damit verbundene Sanktionsmöglichkeiten.

Bringen Arbeitsuchende mit Zielen des Jobcenters divergierende Arbeitsinteressen und Lebensbedürfnisse ohne EGV eher zur Sprache? Wie reagiert das Beratungspersonal auf angesprochene Arbeitsinteressen und Lebensbedürfnisse? Um diese Fragen zu beantworten, werden Beratungen mit und ohne Abschluss der EGV von neu registrierten Leistungsberechtigten verglichen. Methodisch werden Interaktionsbeobachtungen und qualitative Interviews mit Beratungspersonal und Arbeitsuchenden trianguliert und im Anschluss an Erving Goffman ausgewertet.

\section{Institutioneller und theoretischer Hintergrund}

\subsection{Beratungsgespräche im Spannungsfeld zwischen Fördern und Fordern}

Beratung ist ein dialogischer Prozess, der eine gewisse Offenheit voraussetzt. In wechselseitigen Aufgaben- und Rollenzuschreibungen erarbeiten Berater*innen und 
beratene Person eine gemeinsame Problemdefinition sowie Ideen für mögliche Lösungswege (Maier-Gutheil 2016, S. 44). Beratungsgespräche stehen dabei in einem Spannungsfeld. Einerseits sind sie ein Mittel, um Menschen in verschiedenen Lebenslagen $\mathrm{zu}$ helfen, andererseits sind sie durch Machtgefälle geprägt (für einen Überblick siehe McLeod 2013, S. 477 ff.).

Diese Ambivalenz kennzeichnet in besonderem Maße das Beratungsgespräch beim Jobcenter. Es ist ein gesetzlich verankerter Bestandteil des „Förderns“ und soll Leistungsberechtigte bei der Eingliederung in Arbeit unterstützen ( $\$ 14$ SGB II). Gleichzeitig kontrollieren die Fachkräfte die Mitwirkung der Arbeitsuchenden im Sinne des SGB II (Göckler et al. 2014, S. 248). Sie üben damit ein doppeltes Mandat aus: Zu ihren Aufgaben gehört einerseits eine förderliche Unterstützung der Arbeitsuchenden, andererseits jedoch auch die Umsetzung gesetzlicher und Verwaltungsvorgaben, die nicht im Interesse der Arbeitsuchenden liegen müssen (Göckler 2010, S. 264). Auch das Beratungsziel unterscheidet Beratungen im Jobcenter von anderen Beratungen: Es ist gesetzlich vorgegeben. Erwerbsfähige Arbeitsuchende sollen in ihrer Eigenverantwortung ,gestärkt“" werden und eine Arbeit aufnehmen (§ 14 sowie $§ 1$ SGB II). Die dafür nötigen Schritte der Arbeitsmarktintegration sollen Beratungspersonal und Arbeitsuchende in der Beratung gemeinsam erarbeiten.

Typischerweise endet das erste Beratungsgespräch in der Arbeitsvermittlung mit dem gesetzlich vorgeschriebenen EGV-Abschluss. Aufgabe des Beratungspersonals ist, in ihr gemeinsam getroffene Absprachen sowie Teil- und Zwischenschritte zusammenzufassen und die EGV in Folgeterminen gemeinsam mit den Arbeitsuchenden zu überprüfen und fortzuschreiben (§ 15 (3) SGB II, zu einschlägigen Rechtsgrundlagen siehe Banafsche 2013). Rechtlich und institutionell dient die EGV dazu, das Prinzip „Fördern und Fordern“ umzusetzen: In ihr werden zum einen Leistungen für Arbeitsuchende festgelegt, wie die Erstattung von Bewerbungskosten oder eine berufliche Weiterbildung. Zum anderen umfasst sie Bemühungen, die Arbeitsuchende selbst unternehmen und nachweisen müssen, etwa eine festgelegte monatliche Anzahl von Bewerbungen oder die Teilnahme an arbeitsmarktpolitischen Maßnahmen. Dabei ist die EGV ein für Arbeitsuchende wie Jobcenter verbindlicher, öffentlich-rechtlicher Vertrag ( $\S 53$ ff. SGB X). Weist die/der Arbeitsuchende in der EGV festgelegte sog. ,Eigenbemühungen“ nicht nach, stellt dies eine Pflichtverletzung dar und wird mit stufenweisen Mittelkürzungen sanktioniert (§ 31 SGB II). Dabei ist noch offen, ob die infolge eines Bundesverfassungsgerichtsurteils (BVerfG 2019) notwendige Neuregelung im Falle von Pflichtverletzungen zukünftig den EGV-Einsatz verändert. ${ }^{2}$

Die EGV wird als Instrument aktivierender Sozialpolitik breit diskutiert. Oben angesprochene Ambivalenzen des Beratungspersonals in der Arbeitsvermittlung zwischen Unterstützung und Kontrolle finden sich auch in der EGV. Im Unterschied zu einem einseitigen Verwaltungsakt sieht ihr Abschluss ,ein gewisses Maß an Kooperation und Mitsprachechance des Klienten - zumindest theoretisch“ (Weinbach 2012, S. 393) vor. Sie gilt als Chance, feste Ansprüche und damit soziale Rechte

\footnotetext{
2 Das Urteil des Bundesverfassungsgerichts erklärt eine verhältnismäßige Sanktionierung als zulässig, um Mitwirkungspflichten durchzusetzen. Es begrenzt jedoch die Höhe und Dauer von Sanktionen mit Bezug auf die Gewährleistung eines menschenwürdigen Existenzminimums.
} 
aufgrund der Vertragsform zu fixieren (Bieback 2009, S. 189). Kommt jedoch keine einvernehmliche EGV mit Arbeitsuchenden zustande, kann diese von Seiten des Jobcenters per Verwaltungsakt erlassen werden ( $\$ 15$ Abs. 3 SGB II) und ist dann ohne Einigung rechtskräftig. Eine faire Aushandlung der Vertragsinhalte zwischen Arbeitsuchenden und Jobcenter hält die rechtswissenschaftliche Perspektive ,wegen der Möglichkeit der einseitigen Festlegungen [...] für strukturell fraglich“ (Münder 2007, S. 233 f.).

Auch ob Arbeitsuchende ihre Interessen in den EGVs verwirklichen, wird in Frage gestellt. Der Vertrag soll von Arbeitsuchenden und Berater*innen gemeinsam erarbeitet werden. In beobachteten Gesprächen wird er hingegen überwiegend durch das Beratungspersonal vorgegeben (Schütz et al. 2011). Sollte die Eigenverantwortung der Arbeitsuchenden und damit ihre Autonomie betont werden, wird jedoch in der Praxis die Autonomie eingeschränkt (z. B. Ludwig-Mayerhofer et al. 2014, S. 597; Marquardsen 2011; Buestrich et al. 2010). So führt die Machtasymmetrie in der Beratungsinteraktion dazu, dass die EGV weniger als „Vereinbarung“, sondern eher als „Verordnung“ auftritt (Etges und Lenger 2010).

Werden genannte Elemente des Förderns in der EGV in Frage gestellt, sprechen Forschungsergebnisse für die Wirksamkeit des Forderns. Berater*innen nutzen EGVs, um kooperationsunwillige Leistungsberechtigte zu disziplinieren, wie eine Deutungs- und Handlungsmusteranalyse von Arbeitsvermittler*innen zeigt (Behrend et al. 2006, S. 3). Wirksam werden dabei nicht nur tatsächlich verhängte Sanktionen, sondern bereits ihre Existenz und Androhung - etwa im Zuge des EGVAbschlusses. Sie führen zur höheren Konzessionsbereitschaft von Arbeitsuchenden (Götz et al. 2010, S. 3), besitzen jedoch negative Effekte auf den Verdienst nach der Arbeitslosigkeit (van den Berg et al. 2019; Arnie et al. 2013). Weiter bringt eine sanktionsbedingte Leistungskürzung bis unterhalb des soziokulturellen Existenzminimums Betroffene in Problemsituationen. Ob eine frühzeitig geschlossene EGV, die Chancen von Arbeitsuchenden auf einen Arbeitsplatz verbessert, wird uneinheitlich gesehen. Für wenige Arbeitsuchende, die Arbeitslosengeld I beziehen (nach SGB III), fanden van den Berg et al. (2016) positive Effekte. Andererseits können EGV-Inhalte, die nicht ausgehandelt, sondern von den Berater*innen durchgesetzt wurden, zu einer inneren Verweigerungshaltung der Leistungsberechtigten führen (Etges und Lenger 2010, S. 346).

Die bisherige Forschung verweist also auf einen bestehenden, wenn auch ambivalenten Einfluss der EGV auf die Arbeitsvermittlung. Die Befunde sprechen für eine insbesondere disziplinierende Wirkung der EGV bei eingeschränkter Verwirklichung der Autonomie und Mitsprache von Arbeitsuchenden. Ungeklärt ist dabei die Frage, ob Arbeitsuchende ihre Arbeitsinteressen und Lebensbedürfnisse im Beratungsgespräch wirksam einbringen, wenn keine EGV geschlossen und damit verbundene Sanktionsandrohungen nicht möglich sind. Erst die vorliegende Untersuchung kann mittels Variation der Vertragsbeziehungen im Feldexperiment vergleichen, wie fördernde und fordernde Elemente in der Beratung in unterschiedlichen Varianten ausgestaltet werden. 


\subsection{Beratungsgespräche mit Erving Goffman verstehen}

Um zu verstehen, was in der Beratung vor sich geht, nutzt dieser Beitrag die Rahmenanalyse sowie den dramaturgischen Ansatz von Erving Goffman (1977, 2016). Diese Perspektive ermöglicht zu analysieren, wie Arbeitsuchende sowie das Beratungspersonal ihre Persönlichkeit und ihre Ziele in Beratungen interaktiv darstellen.

Treten Beratungspersonal und Arbeitsuchende in dem Beratungsgespräch in Kontakt, definieren sie offen oder unbewusst die Situation, das Gegenüber sowie sich selbst. Sie stellen fest, in welcher Art Beratungssituation sie sind, welche Rollen und Handlungsweisen von ihnen und ihrem Gegenüber erwartet werden und verbinden damit Vorstellungen zur Identität der jeweiligen Person.

Wenn ein Fremder uns vor Augen tritt, dürfte uns der erste Anblick befähigen, seine Kategorie und seine Eigenschaften, seine ,soziale Identität“ zu antizipieren [...], weil persönliche Charaktereigenschaften, wie zum Beispiel „Ehrenhaftigkeit" ebenso einbezogen sind wie strukturelle Merkmale von der Art des „Berufs“(Goffman 1975, S. 10).

Den Eindruck, den Menschen bei anderen erwecken - ihr Image - können Individuen beeinflussen. Goffman (2016) zufolge präsentieren sich Menschen wie Schauspieler*innen in einem Theater. Sie haben ein Ausdrucksrepertoire und inszenieren sich gemäß den antizipierten Erwartungen anderer: Sie spielen eine Rolle und betreiben ,impression management“. Ob ihnen das gelungen ist, sehen sie jedoch erst an der Reaktion ihres Gegenübers. Denn Sprache, Gesten oder statusanzeigende Symbole senden auch entgegen dem eigenen Willen der sich darstellenden Person Informationen, die das Gegenüber nutzen kann, um die Person einzuschätzen und die Authentizität ihrer Darstellung zu prüfen (Goffman 2016). So gesehen ist jedes Zusammentreffen auch ein Kampf gegen die Enttäuschung von Erwartungen, die Verwundbarkeit der Begegnung und Stigmatisierung (Hettlage 2000, S. 163). Unbewusstes Ziel jeglicher Handlung - und damit des ,impression management“ - ist es, Gesicht zu wahren und Schaden an der eigenen Identität zu vermeiden.

E. Goffman fasst die beschriebenen Erwartungen der Interagierenden als Rahmen einer Interaktion. Erfahrungs- und Handlungsschemata lassen Situationen einordnen und ermöglichen angemessenes Handeln (Goffman 1977, S. 274). Kommunikation und das soziale Zusammenleben funktionieren nicht zuletzt, da gesellschaftlich geteiltes Wissen zu Personenkategorien und Handlungsweisen existiert. Diese soziale Ordnung versetzt miteinander agierende Menschen in die Lage, die Erwartungen und Reaktionen des Gegenübers zu antizipieren, ehe sie handeln. Georg Soeffner (2004, S. 143) spricht dabei von implizitem Wissen, anhand dessen Menschen alltägliche Handlungs- und Situationstypen unterscheiden. Sie rekurrieren auf Regeln zur Struktur sozialer Interaktionen, wie Gruß- und Abschiedsrituale, das Vermeiden bestimmter Gesprächsthemen oder eine angemessene Maske (wie Sprechweise, Kleidung). Die Interaktionspartner*innen müssen rahmenadäquat handeln, um die geltenden Regeln und Situationsdefinitionen nicht zu verletzen. 


\section{Daten und Methode}

\subsection{Variation des EGV-Einsatzes in einem Feldexperiment}

Grundlage für die vorliegenden Analysen bildet eine sequenziell explorierende Mixed-Methods-Studie (Creswell 2009) zu einem randomisierten Feldexperiment, das bundesweit in mehreren Jobcentern lief (Bernhard et al. 2019).

Im Rahmen des Feldexperiments wurden neu registrierte Leistungsberechtigte zufällig einer von drei Varianten zugewiesen. In der Standardvariante werden - wie bundesweit üblich - unverzüglich oben dargestellte EGVs abgeschlossen. Diese enthalten Leistungen des Jobcenters, Pflichten des Arbeitsuchenden und eine Rechtsfolgenbelehrung über Kürzungen des Arbeitslosengeldes II (ALG II) bei Nichterfüllung der Pflichten durch die Arbeitsuchenden. Im Feldexperiment werden darüber hinaus zwei weitere Varianten für die ersten sechs Monate eingeführt: EGVs ohne eine Rechtsfolgenbelehrung sowie der Verzicht auf eine EGV. Damit erfolgen in den ersten sechs Monaten in der zweiten und dritten Variante keine Sanktionen auf EGV-Basis. Können Arbeitsuchende also Eigenbemühungen nicht nachweisen oder weigern sich ohne Nennung eines ,wichtigen Grundes“ ( $\$ 31$ SGB II) eine Arbeit oder Ausbildung aufzunehmen oder an arbeitsmarktpolitischen Maßnahmen wie zum Beispiel an Ein-Euro-Jobs (Arbeitsgelegenheit $§ 16 \mathrm{~d}$ SGB II) teilzunehmen, wird das ALG II der Betroffenen nicht stufenweise um $30 \%$, über $60 \%$ des maßgebenden Regelbedarfes bis hin zur vollständigen ALG-II-Zahlungseinstellung bei erneuten Pflichtverletzung innerhalb eines Jahres gekürzt.

Im Feldexperiment wurden damit die finanziell weitreichendsten Sanktionen variiert. Hingegen wurden die anteilig häufigsten Sanktionen infolge von Meldeversäumnissen ausgeschlossen - im Jahr 2019 immerhin 77,4\% (Bundesagentur für Arbeit 2020). Wenn Arbeitsuchende ohne Nennung eines wichtigen Grundes Termine im Jobcenter nicht wahrnahmen, wurde ihr ALG II weiterhin um $10 \%$ des Regelbedarfes für die Dauer von drei Monaten reduziert. In allen Varianten hatten Arbeitsuchende den üblichen Zugang zu Förderleistungen, beschäftigungsfördernden Maßnahmen und zu Beratungsgesprächen.

\subsection{Interaktionsanalysen der Beratungsgespräche}

Dieser Beitrag verortet sich in einer Reihe von Interaktionsanalysen von Beratungsgesprächen in der Arbeitsvermittlung (z. B. Sowa und Staples 2017, 2018; Bernhard 2012) sowie der Sozialen Arbeit (z. B. Schäfer 2019) ebenso wie der Goffman'schen Perspektive (vgl. „Beratungsgespräche mit Erving Goffmann verstehen“). Er vergleicht kommunizierte Ziele von Arbeitsuchenden und Beratungspersonal in der Beratungsinteraktion mit jenen Zielen, die beide Seiten in anschließenden Einzelinterviews benennen.

Daher wurden zwölf vollständige Fälle - bestehend aus Beobachtungen von Beratungsgesprächen und daran anschließende leitfadengestützte Einzelinterviews mit Beratungspersonal und Arbeitsuchenden aus einem umfassenderen Sample (Bernhard et al. 2019, S. 11 f.) ausgewählt. Neun Fälle wurden in zwei der am Feldexperiment teilnehmenden Jobcenter erhoben. Erst- oder Folgeberatungen von unter- 
schiedlichen Arbeitsuchenden wurden in den drei randomisierten Varianten beobachtet. Um die Projektergebnisse beeinflussenden Effekte sozial erwünschter Praktiken im Feldexperiment zu identifizieren und den regulären EGV-Einsatz genauer zu verstehen, wurden die Interaktionsanalysen auch in einem am Feldexperiment unbeteiligten Jobcenter vorgenommen. Davon gehen drei Fälle in die Analyse ein. Die Jobcenter stammen aus drei Regionen, die sich hinsichtlich Siedlungsstruktur und Arbeitsmarktlage unterscheiden. Die Erhebungen fanden von Juli 2017 bis Juni 2018 statt. Beteiligte wurden über die freiwillige Teilnahme und ihre Datenschutzrechte aufgeklärt.

Die teilnehmenden Beobachtungen (für einen Überblick siehe Thierbach und Petschick 2019) fokussierten auf die Kommunikation über Leistungen und Pflichten und wann und wie die EGV zum Einsatz kommt. Die anschließenden leitfadengestützten Interviews (Kruse 2014) behandelten u. a. die verfolgten Ziele, angesprochene Leistungen und Pflichten im Beratungsgespräch. Die Goffman'sche Perspektive dient als analytische Brille für die vorliegende Analyse, hatte auf die Gestaltung der Erhebungsinstrumente jedoch keinen Einfluss. Die transkribierten Audioaufnahmen wurden zunächst inhaltsanalytisch (Mayring 2000) in MAXQDA ausgewertet. Die Kodierung erfasste Ablauf und Reihenfolge der Gesprächsinhalte (z. B. Einstieg, Situationsanalyse, Zielfindung, Leistungen, Pflichten, Gesprächsabschluss). Um Ziele des Beratungspersonals und der Arbeitsuchenden für das beobachtete Beratungsgespräch sowie deren Verhandlung und Darstellung zu analysieren, wurden Transkripte und Beobachtungsprotokolle der Beratungen mit Interviews beider Beteiligter verglichen (Triangulation) und fallvergleichend verdichtet.

\section{Empirische Erkenntnisse zur Beratung im Aktivierungsparadigma}

\subsection{Konvergente und divergente Ziele in den Beratungsgesprächen}

In acht Fällen konvergieren und in vier Fällen divergieren die im Beratungsgespräch vorgebrachten Ziele der Arbeitsuchenden und des Beratungspersonals mit deren genannten Zielen in den Einzelinterviews.

Verfolgen Arbeitsuchende und Beratungspersonal gemeinsam eine Strategie, stimmen sie in Zielen überein, wie dem Abschluss oder der Aufnahme von Sprach-/ Integrationskursen oder beruflichen Weiterbildungen, um in den von beiden Seiten benannten Zielberuf einzumünden. Das Beratungspersonal unterstützt die Arbeitsuchenden beim Abschluss der Qualifikationen mit entsprechenden Freiräumen, z. B. einer geringeren Anzahl an Eigenbemühungen oder späterer Wiedereinladung. In anderen Fällen stehen für beide Seiten die Klärung der Gesundheitssituation sowie das Weiterführen einer niedrigschwelligen Tätigkeit in einer Arbeitsgelegenheit (§ 16d SGB II) im Vordergrund. In einem Fall wird die Arbeitsaufnahme nach Einstellungszusage besprochen.

Hingegen divergieren Ziele, wenn gewünschte Weiterbildungen der Arbeitsuchenden aufgrund unterschiedlicher Zielberufserwartungen nicht ermöglicht werden. Das Beratungspersonal begründet dies im ersten Fall mit einer Arbeitsmarktnachfrage für die bestehende Berufsqualifikation und im zweiten Fall mit einer geringen Arbeits- 
Tab. 1 Verteilung der Fälle ${ }^{\mathrm{a}}$ auf die Kon- oder Divergenz von Zielen zwischen Arbeitsuchenden und Beratungspersonal

\begin{tabular}{llll}
\hline & Ziel-Konvergenz & Ziel-Divergenz & Summe \\
\hline Keine EGV & 3 & 1 & 4 \\
EGV ohne Sanktion & 2 & 1 & 3 \\
Standard-EGV & 1 & 1 & 2 \\
Kein Feldexperiment $^{\mathrm{b}}$ & 2 & 1 & 3 \\
Summe & 8 & 4 & 12 \\
\hline
\end{tabular}

$E G V$ Eingliederungsvereinbarung

${ }^{a}$ Ein Fall besteht aus einer teilnehmenden Beobachtung des Beratungsgespräches sowie Einzelinterviews mit Arbeitsuchenden wie Beratungspersonal.

${ }^{b}$ Fälle wurden in einem am Feldexperiment unbeteiligten Jobcenter erhoben und damit die Standard-EGV. Hingegen wurde in den ersten sechs Monaten die Unterlassung des EGV-Einsatzes sowie der Einsatz einer EGV ohne Sanktionsmöglichkeit und der Standard-EGV in am Feldexperiment teilnehmenden Jobcentern randomisiert.

marktchance des Arbeitsuchenden im gewünschten Beruf. In zwei Fällen benötigen die Arbeitsuchenden zeitliche Freiräume, um für Angehörige zu sorgen und geben an, dem Arbeitsmarkt eingeschränkt zur Verfügung stehen zu können. In allen vier Fällen sprechen Arbeitsuchende die divergierenden Ziele im Beratungsgespräch an.

Bei Zugrundelegung der verschiedenen Varianten lassen sich keine systematischen Verbindungen zu divergierenden oder konvergierenden Zielen rekonstruieren, trotzdem im Sample Fälle für alle Bereiche vertreten sind (Tab. 1).

Mit Blick auf die fordernde EGV-Funktion wäre zu erwarten gewesen, dass Beratungen ohne Sanktionsmöglichkeit auf EGV-Basis weniger disziplinierende Gesprächselemente enthalten und Raum für eine konsensorientierte kooperative Berücksichtigung unterschiedlicher Interessen bieten. In den Fällen ohne EGV (4) oder der EGV ohne Sanktionen (3) sind keine systematisch stärkere Beteiligung der Arbeitsuchenden an der Zielentwicklung oder ein aktiveres Verhandeln der Arbeitsuchenden rekonstruierbar. In den drei Fällen mit Ziel-Konvergenzen ohne eine EGV sind die Übereinstimmungen auf die gemeinsame Zieleinschätzung beider Seiten zurückführen (Einstellungszusage, Weiterbildung, Arbeitsgelegenheit). Doch in zwei Fällen der Ziel-Divergenz aufgrund zu verrichtender Sorgearbeit unterscheiden sich zum einen die Varianten (keine EGV vs. Standard-EGV) sowie das Vorgehen des Beratungspersonals, weshalb deren Analyse im Folgenden dargestellt wird.

\subsection{Darstellung der Fallbeispiele zweier Beratungsinteraktionen im Jobcenter}

Gemeinsam ist den folgenden Fallbeispielen: Die Arbeitsuchenden - nennen wir sie Frau Schmidt und Frau Müller - erklären der jeweils zuständigen Beraterin, dass sie aus familiären Gründen (beide erbringen unbezahlte Sorgearbeit und kümmern sich um Angehörige) dem Arbeitsmarkt nur eingeschränkt zur Verfügung stehen können. Ziel der Beraterin jedoch ist die Vermittlung in Erwerbsarbeit, familiäre Verpflichtungen stehen dahinter zurück. Es wird beobachtet, wie die Beteiligten diese divergenten Ziele verhandeln. 
Gespräch ohne EGV Die 61-jährige Frau Schmidt war bis 1971 in ihrem gelernten Beruf als Modenäherin beschäftigt. Anschließend 15 Jahre als Hausfrau nicht erwerbstätig, machte Frau Schmidt später eine Umschulung und arbeitete in einer Textilreinigung. Nach ihrer Entlassung war sie nicht mehr berufstätig. Aufgrund des Einkommensausfalls ihres erkrankten Lebensgefährten beantragte sie nun ALG-IILeistungen. Beobachtet wird Frau Schmidts zweites Gespräch mit ihrer zuständigen Fachkraft. Im ersten Gespräch wurde aufgrund der Zufallszuweisung im Feldexperiment keine EGV abgeschlossen.

Nach dem Gesprächseinstieg bittet die Beraterin Frau Schmidt am Folgetag Angaben zu ihrer Erwerbsbiografie persönlich nachzureichen. Diese sieht einen Irrtum vorliegen, da sie einer Kollegin der Beraterin die fehlenden Informationen gegeben hätte. Während Frau Schmidt argumentiert und sich rechtfertigt, prüft die Beraterin mithilfe der elektronischen Fallakte, dass ein Gespräch stattfand. Sie klärt Frau Schmidt darüber auf, dass sie sich regional dem Arbeitsmarkt zur Verfügung stellen muss, als Frau Schmidt unvermittelt die Beraterin (B) unterbricht, um ihr Anliegen darzustellen:

Schmidt: Jetzt muss ich mal was dazu [B: Mhm] sagen, weil mein Lebensgefährte ist so schwer krank, ja? [B: Mhm] Ist nur im Krankenhaus und daheim. [B: Mhm] Und am 24. muss er wieder zum Arzt und da lasse ich mir meine Bescheinigung geben, weil ich kann ja nicht arbeiten, ich muss ihn betreuen. $(59: 25)^{3}$

Im Gespräch unterstreicht die Beraterin daraufhin Pflichten der Arbeitsuchenden. Sie verdeutlicht, dass Frau Schmidt für die vollen Grundsicherungsbezüge zeitlich uneingeschränkt verfügbar sein müsse, wenn sie keine Bescheinigung besitzt, dass der erkrankte Lebenspartner eine 24-Stunden-Betreuung benötigt. Im weiteren Gesprächsverlauf fokussiert die Fachkraft die Arbeitsvermittlung und stellt Frau Schmidt zwei Stellenausschreibungen bei Zeitarbeitsfirmen vor, auf die sich die Arbeitsuchende bewerben soll (sog. Vermittlungsvorschläge). Als Frau Schmidt erwähnt, dass sie altersbedingt keine Schichtarbeit übernehmen möchte, erklärt die Beraterin: „Also es geht, das ist das Problem, im Moment nicht darum, was man sich wünscht, sondern das Problem ist, steht dem [der Schichtarbeit - C. F.] irgendetwas entgegen." (50:70) Dann prüft die Beraterin Bewerbungen auf zwei vorherige Vermittlungsvorschläge. Frau Schmidt erklärt, ihr sei nur ein Vermittlungsvorschlag zugesandt worden: Auf den habe sie sich beworben, jedoch keine Antwort erhalten. Eigeninitiativ habe sie sich nicht beworben.

Im Rahmen des Feldexperiments sind keine Leistungskürzungen möglich, wenn sich Frau Schmidt nicht bewirbt. Statt Ziele und Bedürfnisse der Arbeitsuchenden zu klären und zu verhandeln, werden ihre Aussagen hier, wie im weiteren Gesprächsverlauf, mit normverdeutlichenden Gesten der Pflichterfüllung für den Bezug der ALG-II-Leistung gekontert. Ihr Verweis auf die zeitlich uneingeschränkte Arbeitsmarktverfügbarkeit bei vollem Umfang der Grundsicherungsbezüge beinhaltet sogar

\footnotetext{
3 Für eine bessere Lesbarkeit sind die Zitate sprachlich geglättet und Sprechpausenmarkierungen entfernt. In die Auswertung gingen die Originalaussagen ein.
} 
einen indirekten Hinweis auf Kürzungen dieser und damit eine implizite Sanktionsdrohung.

Im Interview mit der Forscherin berichtet die Beraterin, dass sie Frau Schmidt erst in drei Monaten wieder einladen und ihr auch dann einen „leichten Puffer“ lassen möchte, wenn sie Vollzeit dem Arbeitsmarkt zur Verfügung stehen müsste, dann „haben beide das Gesicht gewahrt“ (61:61). In ihrer Haltung zum weiteren Vermittlungsprozess geht sie auf die Erwartungen der Arbeitsuchenden bezüglich der Freiräume für die Sorgearbeit ein und zeigt sich kooperativ. Auf der anderen Seite reagiert sie im beobachteten Gespräch disziplinierend: Weder recherchiert sie Stellen ohne Schichtarbeit, noch Teilzeit-Angebote, falls Frau Schmidt mehr Zeit für die Pflege ihres Mannes aufwenden muss (61:51). Mit diesem Handeln bekräftigt sie Elemente des Forderns durch normverdeutlichende Aussagen und eine implizite Sanktionsdrohung. Im Kontrast dazu steht das folgende Gespräch.

Gespräch mit EGV Die etwa 50-jährige Frau Müller verfügt über langjährige Erfahrung im Verkauf und leitete zuletzt einen Imbiss. Aufgrund einer chronischen Erkrankung war sie zunächst arbeitsunfähig und ist seit über drei Jahren nicht mehr berufstätig. Sie erscheint zum dritten Gespräch mit ihrer zuständigen Beraterin. Sie steht in einer Vertragsbeziehung, denn im Erstgespräch schloss sie eine EGV mit Rechtsfolgebelehrung und Sanktionsmöglichkeit mit dem Jobcenter ab.

Nach der Begrüßung fragt die Beraterin Frau Müller nach dem Ausgang eines Vorstellungsgespräches, woraufhin sich ein Gespräch über im Ergebnis bislang erfolglose, jedoch zahlreiche Bewerbungsaktivitäten ergibt. Im Gespräch verweist Frau Müller auf die in diesem Zuge erstellten Bewerbungsunterlagen, die sie in einem Ordner mit sich führt. Nach dieser Klärung berichtet Frau Müller von ihrem Anliegen:

Müller: Ich habe nur im Moment ein kleines Problem, seit einem Monat liegt meine Mutti im Sterben und ich renn nur noch ins Krankenhaus und [B: Mhm] da ist bisschen, sag ich mal.

B: Persönlich ein bissel auch angegriffen.

Müller: Ja.

B: Ja.

Müller: Die hat nicht mehr lang. Die hat Leberzirrhose im Endstadium. [B: Mhm] Und da habe ich einige Probleme zurzeit. [B: Mhm] Ich sag mal, nicht so viel Gedanken frei, dass ich sagen könnte, ich kann mich von früh bis abends [B: Mhm] kümmern, dass ich was [B: Mhm] finde. Ne? [B: Okay] (50:46-51)

Das folgende Gespräch ist von einer Aushandlung der Möglichkeiten beider Seiten geprägt. Die Beraterin nimmt die aktuell anstehende Sorgearbeit von Frau Müller nicht nur empathisch auf, sondern verschiebt die Erfassung von Frau Müllers Bewerbungsaktivitäten im digitalen System und flexibilisiert ihre Teilnahme an einer berufsqualifizierenden Maßnahme für den konvergenten Zielberuf Verkauf. Dies begründet die Beraterin im Interview mit der Forscherin damit, dass Frau Müllers persönliche Einschränkungen nachvollziehbar seien und ,es bringt auch nichts, weil komm ich ja letztendlich auch nicht weiter“ (52:21). Im Gespräch klärt Frau Müller 
den Handlungsspielraum ihrer Beraterin mit der Nachfrage: „Ich meine, man darf ja nicht jeden Tag ins Krankenhaus [B: Mhm], das ist es ja.“ (50:79) Im aktuellen Vorgehen kann die Beratungsfachkraft nachweisen, dass sie Frau Müller in eine Qualifizierungsmaßnahme vermittelt hat und sie gesetzeskonform dem Arbeitsmarkt zur Verfügung steht. Die Aushandlung ermöglicht dabei Frau Müller weiterhin Krankenhausbesuche, da sie ihre Maßnahmenteilnahme absagen kann. Der Maßnahme steht sie nach 50 Jahren Berufserfahrung jedoch skeptisch gegenüber: „Ich setz mich da gerne rein, aber ob es was bringt?" (51:31)

Bei dem Vermittlungsvorschlag einer Teilzeitstelle in einer Bäckerei (mit zwei Schichten) verhandelt Frau Müller über zaghafte Ansprache der Entfernung und Bezahlung (,Dann zahlen sie bloß den Mindestlohn und dann hab ich am Ende durch den Spritverbrauch nichts“ 50:138). Die Beraterin kommt zu dem Schluss: „Lassen wir das mal“ (50:139). Die Beraterin insistiert nicht auf diese Stellenausschreibung, sondern übt ihr Ermessen orientiert an den vorgetragenen Zielen der Arbeitsuchenden aus.

Auch diese Beratungssituation enthält disziplinierende Elemente, die das Ziel der Arbeitsmarktintegration verdeutlichen und Frau Müller dazu bewegen, einer Qualifizierungsmaßnahme zuzustimmen, deren Informationsgewinn und Vermittlungserfolg sie gering einschätzt. Die bestehende EGV wird nicht explizit angesprochen oder interaktiv eingebunden. Nichtsdestotrotz werden die Ziele beider Interagierenden konsensorientiert berücksichtigt, bei Wahrung des Images beider Seiten. Frau Müller werden Freiräume für die Sorgearbeit eingeräumt und sie erhält einen anderen Vermittlungsvorschlag, als sie Bedenken anklingen lässt. Gleichzeitig legt die Beraterin mit Frau Müller nachweisbar gegenüber Vorgesetzten weitere Schritte für die Arbeitsmarktintegration fest. Im Grunde erscheint diese Beobachtung als kooperative Verhandlung, in der sich beide Seiten respektieren.

\subsection{Impression Management - zwischen Normbruch und Konformität}

Die Interessen der Arbeitsuchenden sind in obigen Beratungssituationen gleich: Sie wollen sich um Angehörige kümmern und befinden sich im Konflikt mit den Anforderungen der Grundsicherung, dem Arbeitsmarkt umfassend zur Verfügung zu stehen. Frau Müller steht in einem Vertragsverhältnis mit dem Jobcenter, ihre Beratung ist allerdings geprägt von einer Aushandlung und einem Interessenausgleich zwischen ihr und der Beraterin. Frau Schmidts Beratung hingegen enthält zwar keine EGV, aber deutlich disziplinierende Elemente (Stellenauswahl, Normverdeutlichung, implizite Sanktionsdrohung). Dabei lassen sich Unterschiede rekonstruieren, welche Wirkungen die Selbstdarstellungen der Arbeitsuchenden bei den jeweiligen Beraterinnen erzielen.

In ihrer Selbstdarstellung versucht Frau Schmidt zu demonstrieren, dass sie Mitwirkungspflichten nachkommt. Andererseits kommuniziert sie Einschränkungen ihrer Arbeitsbereitschaft offen (Sorgearbeit, Schichtarbeit) und latent (fehlende Initiativbewerbungen). Für die Beraterin mehren sich im Gesprächsverlauf Zeichen, dass Frau Schmidt die Normen der Pflichterfüllung bricht: Frau Schmidt habe sich weder gemerkt, den Lebenslauf zu vervollständigen, noch wolle sie sich auf Vermittlungsvorschläge bewerben und grundsätzlich wolle sie nicht arbeiten. Die Möglichkeit 
technischer oder institutioneller Kommunikationsschwierigkeiten bei der Vervollständigung der Erwerbsbiografie in der elektronischen Fallakte oder des nichtübermittelten Vermittlungsvorschlages zieht sie nicht in Erwägung. Ihr Bild von Frau Schmidt prägt: „sie war ja Hausfrau und ihr Lebensgefährte ist selbstständig, und weil er nun jetzt nicht selbstständig tätig ist, da müssen sie was machen wegen dem Geld. Aber das Wort Arbeit kam da auch nicht vor.“ (61:27) So deutet die Beraterin Frau Schmidts Verhalten als mangelnde Motivation und Konzessionsbereitschaft.

Auch einige Reaktionen von Frau Müller hätten als Zeichen der Demotivation gedeutet werden können, etwa als sie verhalten Stellenausschreibungen kommentiert. Weiter waren Frau Müllers Bewerbungsbemühungen im Ergebnis auch nicht erfolgreicher, sie berichtet von Absagen und Eingangsbestätigungen. Frau Müller entspricht jedoch den Konformitätserwartungen ihrer Beraterin und wird als durchgängig erwerbstätige Person beschrieben, die aufgrund einer Erkrankung ihren Job verlor. Sie berichtet nicht nur von mehreren (auch eigeninitiativen) Bewerbungen, sondern zeigt angesprochene Briefe und Bewerbungen unaufgefordert vor. Dabei besitzt Frau Müller mit Blick auf die divergierenden Ziele günstigere Ausgangsbedingungen: Ihre unbezahlte Sorgearbeit erscheint temporär begrenzter und sie verfügt bereits über ein ärztliches Attest, das ihr eine eingeschränkte Arbeitsmarktfähigkeit dokumentiert.

Weiter prägen gesellschaftliche Ungleichheitsverhältnisse das Bild der aktiven erwerbstätigen Frau Müller und der inaktiven „Hausfrau“ Frau Schmidt: Sorgearbeit, wie Hausarbeit, die Pflege von Kindern und älteren Angehörigen oder ehrenamtliche Tätigkeit, erfährt durch die fehlende Entlohnung eine geringere gesellschaftliche Anerkennung als Lohnarbeit. Laut OECD-Statistik erbringen Frauen fast doppelt so viel Zeit in unbezahlter Arbeit (263 min. am Tag) als Männer (OECD 2021). Aktivierende Sozialpolitik zielt dabei auf eine stärkere Erwerbsbeteiligung von Frauen, eine Gleichstellung von Frauen und Männern setzt jedoch die Gleichverteilung von bezahlter und unbezahlter Arbeit voraus (Pascall und Lewis 2004).

Die Analysen weisen darauf hin, dass das Beratungspersonal Arbeitsuchende in der alltäglichen Arbeit im Jobcenter in motivierte und demotivierte Arbeitsuchende unterscheidet. Dies bestätigt auch die bundesweite Online-Befragung des Beratungspersonals in sieben Jobcentern: $75 \%$ der Berater*innen schätzen den EGV-Einsatz bei demotivierten Personen als sinnvoll/eher sinnvoll ein, entsprechend $60 \%$ bei motivierten Personen als nicht/eher nicht sinnvoll (Bernhard et al. 2019, S. 19). So spricht sich das befragte Beratungspersonal für Instrumente aus, um gegenüber Arbeitsuchenden Pflichten durchsetzen zu können, diese jedoch im Bedarfsfall im Rahmen ihres Ermessens abzumildern, was folgende Aussage illustriert:

B: Aber die müssen ja auch merken, dass sie hier in einer gewissen Pflicht sind. Also, dass eben Arbeitslosengeld II nicht nur Recht heißt, sondern auch Pflicht heißt. Und (...) ich bin bestimmt einer der Vermittler, (...) die auf viele Sachen Rücksicht nehmen. (...) Wo ich dann immer noch sag, wenn der mir irgendwas Tragfähiges sagen kann, warum er nun da gerade nicht kommt, dann tu ich das manchmal schon ein bisschen mit abbiegen, was dann nicht so ganz dem Gesetz entspricht. (...) Aber wenn jetzt einer nun so gar nicht möchte, ne? (61:51) 
Das vorliegende Material lässt den Schluss zu, dass das Entgegenkommen des Beratungspersonals neben der Nennung triftiger Gründe bei Pflichtverletzungen durch das ,impression management“ Arbeitsuchender mitbedingt ist. Berater*innen prüfen anhand der Bewerbungsbemühungen sowie der Selbstdarstellung der Arbeitsuchenden, ob diese motiviert sind, eine Erwerbstätigkeit aufzunehmen. Arbeitsuchende wissen um die Bedeutung der Selbstdarstellung im Beratungsprozess, was folgendes Zitat einer anderen Arbeitsuchenden verdeutlicht: ,dass die auch sehen, man hat selbst irgendwie das Interesse daran." (38:47) Äußerungen und Handlungen, die bei motiviert geltenden Personen als kleines Versehen oder auch als Missverständnis interpretiert werden können, dienen bei Frau Schmidt dazu, das Bild einer demotivierten Arbeitsuchenden zu bestätigen. Das beinhaltet die Gefahr, dass Arbeitsuchende, die ihnen einmal zugeschriebene Rolle der/des Demotivierten nicht ablegen können. Bereits Goffman stellte fest, dass Arbeitsuchende „Willensschwäche, beherrschende oder unnatürliche Leidenschaften, tückische und starre Meinungen und Unehrenhaftigkeit" (Goffman 1975, S. 12f.) zugeschrieben werden. Die Zuschreibung wird dann zur selbsterfüllenden Prophezeiung, wenn Abwehrreaktionen der Arbeitsuchenden wiederum als Symptome für die Demotivation gedeutet werden.

\subsection{Rahmenadäquates Handeln im Aktivierungsparadigma}

Weiter unterscheiden sich die Arbeitsuchenden hinsichtlich des Zeitpunktes, wann sie ihre Sorgearbeit gegenüber dem Beratungspersonal ansprechen: Frau Schmidt äußert ihr Anliegen zur eingeschränkten Verfügbarkeit für den Arbeitsmarkt bevor, hingegen Frau Müller nachdem die (eigenen) Vermittlungsschritte abgearbeitet worden waren.

Die Analyse der zwölf beobachteten Beratungsgespräche bestätigt, dass Vermittlungsgespräche einer recht stabilen Dramaturgie folgen (Sowa und Staples 2013). Nach der Begrüßung folgt typischerweise die Klärung der Bewerbungsaktivitäten, ggf. eine Anpassung der Arbeitsmarktintegrationsstrategie, die Verhandlung und Ausgabe von Vermittlungsvorschlägen, und anschließend ist Raum für Anliegen der Arbeitsuchenden. Daher liegt der Schluss nahe, dass Frau Schmidts Darstellung des eigenen Anliegens vor der Besprechung ihrer Mitwirkungspflichten ihr Image beeinträchtigte. Hingegen beförderte Frau Müllers Image, dass sie zur rechten Zeit ihr Anliegen anspricht, unaufgefordert Bewerbungsbemühungen nachweist und ihre Interessen bei Klärung des Handlungsspielraums der Beraterin verhandelt. Sie handelt mit Goffman gesprochen rahmenadäquat - entsprechend der Interaktionsregeln der Beratung im Jobcenter.

Mit Perspektive des Goffman'schen dramaturgischen Ansatzes (2016) „spielen“ Berater*innen und Arbeitsuchende ein „Theaterstück Beratung“. Das Beratungspersonal ist mit dem Stück vertraut und dirigiert es. Die gelebten Routinen, das implizite Wissen (Soeffner 2004, S. 143) sowie ihre Position als Behördenvertreter*innen verleihen ihnen die Rolle des Regieführenden. Mit McLeod gesprochen, kennt das Beratungspersonal die ,rules of the game“ (McLeod 2013, S. 473). Damit verfestigt sich die ungleiche Verhandlungsposition zwischen Arbeitsuchenden und Beratungspersonal - neben der unterschiedlichen Verteilung von Fach- und Methodenwissen (Schütz et al. 2011; Göckler 2010; Hielscher und Ochs 2009). In diesem 
Stück ist die EGV eine Requisite, die das Prinzip Fördern und Fordern symbolisiert und im Rahmen der Gesprächsführung mit unterschiedlicher Bedeutung belegt werden kann - etwa als „Zusammenfassung“ oder „Vertrag“ (Freier 2021). Sie legt die Integrationsziele fest und ist zugleich eine Regieanweisung für die nächsten Beratungsinteraktionen, in denen die Einhaltung der Pflichten geprüft und Förderungen besprochen werden.

Disziplinierung im Aktivierungsparadigma beinhaltet dabei das Risiko, dass Arbeitsuchende in den gegebenen Interaktionsregeln strategische Geheimnisse gegenüber dem Beratungspersonal entwickeln, um ihr Image nicht zu gefährden. Verschärfte Zumutbarkeitskriterien für die Arbeitsaufnahme von Arbeitsuchenden befördern Interessendivergenzen von Arbeitsuchenden und Jobcenter. Beispielsweise bewarb sich Frau Müller erfolgreich bei einem für sie wenig attraktiven Vermittlungsvorschlag des Jobcenters. Doch im Vorstellungsgespräch wies sie auf gesundheitliche Einschränkungen hin (Als-ob-Bewerbung, vgl. auch Etges und Lenger 2010). Dabei hätte sie den Vermittlungsvorschlag legitim durch Vorlage ihres ärztlichen Attestes zurückweisen können. Im gegebenen Beratungssetting schreibt Frau Müller sich wenig Autonomie und Handlungsmacht zu, um ihre Arbeitsinteressen einzubringen: „Ich muss es ja machen. Was soll ich machen? Ich finde es aber schade." (51:27)

Die Rolle motivierte respektive demotivierte Arbeitsuchende schreiben Berater*innen ihren Adressat*innen in der Interaktion zu. Dabei befördern implizites Wissen zu Interaktionsregeln der Beratung im Jobcenter sowie die der Pflichterfüllung konforme Selbstdarstellung ein gelungenes ,impression management“ Arbeitsuchender.

\section{Resümee}

Beratung im Jobcenter unterscheidet sich von anderen Beratungssituationen u.a. durch den Abschluss einer sanktionierbaren EGV. Bestehende Forschung verweist auf einen existierenden, wenn auch ambivalenten Einfluss der EGV als Instrument aktivierender Sozialpolitik. Rechtslage und Praxis der EGV werden insbesondere als Betonung von fordernden Elementen sowie einer geringen Offenheit für Bedürfnisse und Autonomie der Arbeitsuchenden kritisiert (z. B. Weinbach 2012; Marquardsen 2011; Bieback 2009). Ob Arbeitsuchende ihre Arbeitsinteressen und Lebensbedürfnisse in anderer Form einbringen, wenn keine EGV geschlossen und damit verbundene Sanktionen nicht möglich sind, untersucht dieser Beitrag empirisch. Mittels Variationen der Vertragsbeziehungen von Arbeitsuchenden und Jobcenter konnte im Rahmen eines Feldexperiments verglichen werden, wie fördernd respektive fordernd Beratung in den unterschiedlichen Varianten ausgestaltet wird. Dafür wurden zwölf Beratungsinteraktionen mit Interviewdaten von Arbeitsuchenden und Beratungspersonal trianguliert.

Die Bedeutung von Zielen sowie Interaktionsregeln im Beratungsgespräch wurden mit Rückgriff auf Erving Goffman (1977, 2016) anhand zweier Fallbeispiele mit und ohne EGV betrachtet. Mit Blick auf die disziplinierende EGV-Funktion wäre zu erwarten gewesen, dass die Beratung ohne EGV weniger disziplinieren- 
de Gesprächselemente enthält und Raum für eine konsensorientierte kooperative Berücksichtigung unterschiedlicher Interessen bietet. Die Interaktionsbeobachtungen zeigen jedoch, dass für das Beratungsgespräch weniger relevant war, ob Arbeitsuchende durch eine EGV in einer rechtsverbindlichen Vertragsbeziehung zum Jobcenter standen. Um Elemente des Forderns umzusetzen, wurde die EGV durch sprachliche Normverdeutlichung der Pflichterfüllung bis hin zu impliziter Sanktionsdrohung substituiert. Hingegen beeinflusst die Rahmung der Gesprächssituation - zum einen durch das Beratungspersonal und zum anderen durch das Aktivierungsparadigma -, ob es einen eher disziplinierenden oder kooperativen Charakter besitzt.

Ob Arbeitsuchende sich mit ihren gesamten Kompetenzen und Förderbedarfen in das Beratungsgespräch einbringen können sowie die daran ansetzenden Beschäftigungsmaßnahmen, hängen nicht nur vom individuellen Beratungsstil (Rübner 2018) oder dem Gesprächsmodus der Fachkräfte (Böhringer und Karl 2012, S. 290) ab. Das Beratungspersonal orientiert seine Gesprächsführung daran, ob es Arbeitsuchende als motiviert und aktiv einschätzt. Arbeitsuchende müssen dokumentieren und im Gespräch demonstrieren, dass sie sich bewerben. Bedeutend ist damit, inwiefern den Arbeitsuchenden ein ,impression management“ als motivierte und aktive Arbeitsuchende gelingt. Das erhärtet die Ergebnisse einer ethnografischen Studie in Jobcentern, dass für den guten Eindruck beim Beratungspersonal auch bedeutend ist, dass Arbeitsuchende ihre Folgsamkeit demonstrieren (Grimmer 2018). So engagiert das Beratungspersonal in Jobcentern auch ist, strukturell erleichtert es ihnen ihre Arbeit, wenn Arbeitsuchende mit allen Aspekten des Beratungspersonals einverstanden sind und koproduktive Chancen im Beratungsprozess nicht nutzen. Ergreifen die Arbeitsuchenden hingegen Verwirklichungschancen und versuchen sie, ihre Arbeitsinteressen durchzusetzen, riskieren sie, das Bild einer/eines nichtmotivierten Arbeitsuchenden zu verstärken. Andererseits kann das Wissen um ein passendes „,impression management“" es ermöglichen, Arbeitsuchende zu trainieren, einen motivierten Eindruck zu hinterlassen. In anderen Handlungsfeldern hat sich bereits gezeigt, dass sozialpädagogische Beratung solche Erkenntnisse nutzen kann (Lochner und Bastian 2018, S. 292). Dabei ist selbst kooperativen Beratungsgesprächen im Aktivierungsparadigma Disziplinierung eingeschrieben. $\mathrm{Zu}$ dem asymmetrischen Machtverhältnis in der Arbeitsvermittlung tritt, mit Foucault gesprochen, ein wirkmächtiger Machtdiskurs hinzu, der legitimes Wissen und Werte mit Regierungshandeln verbindet (1991, 2004; für eine Analyse des Diskurses siehe Freier 2016, S. 47 ff., 2018). Im Kontext solcher Normierungen gestalten Subjekte ihr Selbst und ihre Lebensweise auch mittels Selbstdisziplinierungstechniken: Im Fall von Frau Müller kommen Arbeitsinteressen nicht zur Sprache, um das Image einer motivierten Arbeitsuchenden zu bewahren. Der fehlende Informationsaustausch behindert passgenauere Stellenvermittlungen, und der Vermittlungsvorschlag geriert hier zum Selbstzweck. Außerdem besteht das Risiko, dass Arbeitsuchende ihre Verwirklichungschancen in der Beratung nicht nutzen, selbst wenn die Berater*innen eine kooperative Haltung einnehmen.

Erwähnt werden sollen Limitationen der vorliegenden Untersuchung. Da Sanktionsmöglichkeiten einzig auf EGV-Basis zeitweise im Feldexperiment außer Kraft gesetzt werden konnten, wurden zwar die finanziell weitreichendsten jedoch nicht 
alle Sanktionsmöglichkeiten variiert. Zudem waren Arbeitsuchenden sanktionierbare Pflichten auch dann bekannt, wenn sie in keinem Vertragsverhältnis zum Jobcenter standen. Ebenfalls ist den Arbeitsuchenden der Aspekt des Forderns präsent, zum Beispiel, wenn sie die Termineinladung als „Vorladung“ interpretieren (Gurr et al. 2018, S. 237). Daher können die vorliegenden Ergebnisse keinen Hinweis darauf geben, wie sich die Beratungspraxis bei grundlegenderen sozialpolitischen Veränderungen umgestalten würde.

Was also tun, um die Arbeitsvermittlung stärker an den Arbeitsinteressen zu orientieren und Lebensbedürfnisse und -kompetenzen anzuerkennen? Die EGV von Sanktionsmöglichkeiten zu entkoppeln, kann ein erster Schritt sein (Senghaas et al. 2020; Bernhard et al. 2019). Das hier dargestellte Material lässt jedoch darauf schließen, dass dies nicht ausreicht, um eine konsensorientierte und kooperative Beziehung zwischen Arbeitsuchenden und dem Beratungspersonal zu festigen oder die Autonomie der Arbeitsuchenden zu stärken. Vielmehr zeigen die Befunde, dass es notwendig ist, die Verhandlungsposition der Arbeitsuchenden zu stärken, etwa über grundsätzliche Mitspracherechte und substanzielle Rechtsansprüche auf Eingliederungsleistungen (Betzelt und Bothfeld 2014, S. 4) oder Beratungsgespräche vor dem EGV-Abschluss (Etges und Lenger 2010, S. 350). Mit Blick auf Frau Müllers Fallbeispiel könnte ein vorab informierendes Beratungsgespräch beitragen, Defizite an Informationen (Schütz et al. 2011) abzubauen. Das implizite Wissen zu Regeln im Umgang mit dem Beratungspersonal der Arbeitsvermittlung hingegen kann nicht durch häufigere Beratung erlernt werden. Zudem bestehen Hinweise, dass dieses implizite Wissen zum Umgang mit dem Beratungspersonal in sozial benachteiligten Bevölkerungsschichten weniger verbreitet ist. Denn Menschen mit geringerem oder ohne Schulabschluss mangelt es an Wissen zu erfolgreichem Handeln im Jobcenter (Zahradnik et al. 2016). Sie verfügen über ein geringeres kulturelles Kapital und stehen dem Beratungspersonal aufgrund der unterschiedlichen sozialen Herkunft und sozialen Position (siehe für die Arbeitsvermittlung auch Ludwig-Mayerhofer et al. 2014; für die Beratung allgemein McLeod 2013, S. 485 ff.) in einer habituellen Distanz gegenüber. Diese Faktoren erhöhen auch das Sanktionsrisiko der sozial Benachteiligten (Zahradnik et al. 2016). Schwierigkeiten der Arbeitsuchenden jenseits ihrer sozialen und gesellschaftlichen Basis zu betrachten, kann dazu führen, diese Schwierigkeiten in unangemessene Hilfsmuster einzupassen und Konflikte als Beziehungsprobleme zwischen Beratungspersonal und ihrem Gegenüber zu interpretieren (Thiersch 2013, S. 213). Berater*innen in der Arbeitsvermittlung wie auch die Sozialpolitik sind gefordert, solche Mechanismen zu reflektieren, um soziale Ungleichheitsstrukturen nicht zu festigen.

Danksagung Ich danke Sarah Bernhard, Stefan Bernhard, Martina Pistor, Ina von Seckendorff und den zwei unbekannten Begutachtenden für wertvolle Hinweise sowie Ulrike Büschel und Maren Müller für die Unterstützung in der Analyse.

Funding Open Access funding enabled and organized by Projekt DEAL.

Open Access Dieser Artikel wird unter der Creative Commons Namensnennung 4.0 International Lizenz veröffentlicht, welche die Nutzung, Vervielfältigung, Bearbeitung, Verbreitung und Wiedergabe in jeglichem Medium und Format erlaubt, sofern Sie den/die ursprünglichen Autor(en) und die Quelle ordnungsgemäß nennen, einen Link zur Creative Commons Lizenz beifügen und angeben, ob Änderungen vorgenommen wurden. 
Die in diesem Artikel enthaltenen Bilder und sonstiges Drittmaterial unterliegen ebenfalls der genannten Creative Commons Lizenz, sofern sich aus der Abbildungslegende nichts anderes ergibt. Sofern das betreffende Material nicht unter der genannten Creative Commons Lizenz steht und die betreffende Handlung nicht nach gesetzlichen Vorschriften erlaubt ist, ist für die oben aufgeführten Weiterverwendungen des Materials die Einwilligung des jeweiligen Rechteinhabers einzuholen.

Weitere Details zur Lizenz entnehmen Sie bitte der Lizenzinformation auf http://creativecommons.org/ licenses/by/4.0/deed.de.

\section{Literatur}

Arnie, P., Lalive, R., \& van Ours, J.C. (2013). How effective are unemployment benefit sanctions? Looking beyond unemployment exit. Journal of Applied Econometrics, 28(7), 1153-1179.

Banafsche, M. (2013). Die Eingliederungsvereinbarung zwischen Subordination und Koordination - Ausdruck eines alten verwaltungsrechtlichen Diskurses. Soziales Recht, 3(4), 121-139.

Behrend, O., Ludwig-Mayerhofer, W., Sondermann, A., \& Hirseland, A. (2006). Reform der Arbeitsverwaltung: Im Schatten der Aufmerksamkeit - die Arbeitsvermittler. IAB-Kurzbericht, 21/2006. Nürnberg: Institut für Arbeitsmarkt- und Berufsforschung.

van den Berg, G., Hofmann, B., Stephan, G., \& Uhlendorff, A. (2016). Eingliederungsvereinbarungen in der Arbeitslosenversicherung. Nur ein Teil der Arbeitslosen profitiert von frühen Abschlüssen. IABKurzbericht 3/2016. Nürnberg: Institut für Arbeitsmarkt- und Berufsforschung.

van den Berg, G., Uhlendorff, A., \& Wolff, J. (2019). The impact of sanctions for young welfare recipients on transitions to work and wages and on dropping out. Working Paper, No. 2019:5. Uppsala: Institute for Evaluation of Labour Market and Education Policy (IFAU).

Bernhard, S. (2012). Selbstständige in der Arbeitsverwaltung: Eine Rahmenanalyse nach Goffman. In M. Bereswill, C. Figlestahler, L. Y. Haller, M. Perels \& F. Zahradnik (Hrsg.), Wechselverhältnisse im Wohlfahrtsstaat: Dynamiken gesellschaftlicher Justierungsprozesse (S. 233-254). Münster: Westphälisches Dampfboot.

Bernhard, S., Senghaas, M., Freier, C., Ramos Lobato, P., \& Stephan, G. (2019). Vertragsbeziehungen zwischen Jobcentern und Arbeitslosen: Eingliederungsvereinbarungen aus Sicht von Arbeitsvermittlerinnen und Arbeitsvermittlern. IAB-Forschungsbericht, 2/2019. Nürnberg: Institut für Arbeitsmarktund Berufsforschung.

Betzelt, S., \& Bothfeld, S. (2014). Autonomie - ein neues Leitbild einer modernen Arbeitsmarktpolitik. WISO-direkt Oktober 2014. Bonn: Friedrich-Ebert-Stiftung.

Bieback, K.-J. (2009). Kooperation im Zwangsverhältnis. Teilhaberechte und Vertragsstrukturen in der Arbeitsmarktverwaltung. Zeitschrift für Rechtssoziologie, 30(2), 185-213.

Böhringer, D., \& Karl, U. (2012). Gestalt und Gestaltung von Gesprächen in Jobcentern und der Berufsberatung. пеие praxis, 12(3), 274-294.

Buestrich, M., Dahme, H.-J., Kühnlein, G., \& Wohlfahrt, N. (2010). Funktionale Professionalisierung. Die Betreuung der Überflüssigen und ihre sozialarbeitsbezogenen Konsequenzen. In H. Burghardt \& R. Enggruber (Hrsg.), Soziale Dienstleistungen am Arbeitsmarkt in professioneller Reflexion Sozialer Arbeit (S. 237-255). Berlin: Frank \& Timme.

Bundesagentur für Arbeit (2020). Sanktionen: Zeitreihe Monats- und Jahreszahlen seit 2007. Nürnberg: Bundesagentur für Arbeit.

BVerfG (2019). Urteil des Ersten Senats. Az: 1 BvL 7/16, Rn. 1-225, 1-225. http://www.bverfg.de/e/ 1s20191105_1bv1000716.html. Zugegriffen: 3. Jan. 2021.

Creswell, J.W. (2009). Research design: qualitative, quantitative and mixed methods approaches. Thousand Oaks: SAGE.

Dahme, H.-J., Trube, A., \& Wohlfahrt, N. (2008). Soziale Arbeit für den aktivierenden Staat. In Bielefelder Arbeitsgruppe 8 (Hrsg.), Soziale Arbeit in Gesellschaft (S. 268-275). Wiesbaden: Springer VS.

Etges, M.S., \& Lenger, A. (2010). Die Eingliederungsvereinbarung des SGB II. Eine kritische Betrachtung aus ordnungsökonomischer Perspektive. Zeitschrift für Wirtschaftspolitik, 59(3), 329-356.

Foucault, M. (1991). Die Ordnung des Diskurses. Frankfurt a. M.: Fischer.

Foucault, M. (2004). Die Geburt der Biopolitik. Geschichte der Gouvernementalität, Bd. 2. Frankfurt a. M.: Suhrkamp.

Freier, C. (2016). Soziale Aktivierung von Arbeitslosen? Praktiken und Deutungen eines neuen Arbeitsmarktinstruments. Bielefeld: transcript. 
Freier, C. (2018). Hoffnung im aktivierenden Sozialstaat? Sozialpolitische Dispositive in der Arbeitsverwaltung. In S. Betzelt \& I. Bode (Hrsg.), Angst im neuen Wohlfahrtsstaat. Kritische Blicke auf ein diffuses Phänomen (S. 295-317). Baden-Baden: Nomos.

Freier, C. (2021). Zusammenfassung oder Vertrag? Wie Vermittlungsfachkräfte im Jobcenter die Eingliederungsvereinbarung einsetzen. Blätter der Wohlfahrtspflege, 167(1), 33-35.

Galuske, M., \& Rietzke, T. (2005). Aktivierung und Ausgrenzung. Aktivierender Sozialstaat, Hartz-Reformen und die Folgen für Soziale Arbeit und Jugendberufshilfe. In R. Anhorn, F. Bettinger \& J. Stehr (Hrsg.), Sozialer Ausschluss und Soziale Arbeit: Positionsbestimmungen einer kritischen Theorie und Praxis Sozialer Arbeit (S. 399-416). Wiesbaden: Springer VS.

Göckler, R. (2010). Sanktionsgespräche in der Grundsicherung für Arbeitsuchende (SGB II) und ihre Anschlussfähigkeit zu Beratungsstandards. Sozialer Fortschritt, 59(10/11), 264-272.

Göckler, R., Rübner, M., Kohn, K.-H.P., Jäger, U., \& Franck, M. (Hrsg.). (2014). Beschäftigungsorientiert beraten und vermitteln. Regensburg: Walhalla.

Goffman, E. (1975). Stigma: Über Techniken der Bewältigung beschädigter Identität. Frankfurt a. M.: Suhrkamp.

Goffman, E. (1977). Rahmenanalyse. Ein Versuch über die Organisation von Alltagserfahrungen. Frankfurt am Main: Suhrkamp.

Goffman, E. (2016). Wir alle spielen Theater. Die Selbstdarstellung im Alltag. München, Berlin: Piper.

Götz, S., Ludwig-Mayerhofer, W., \& Schreyer, F. (2010). Sanktionen im SGB II: Unter dem Existenzminimum. IAB-Forschungsbericht, 10/2010. Nürnberg: Institut für Arbeitsmarkt- und Berufsforschung.

Grimmer, B. (2018). Folgsamkeit herstellen: Eine Ethnographie der Arbeitsvermittlung im Jobcenter. Bielefeld: transcript.

Gurr, T., Unger, S., \& Jungbauer-Gans, M. (2018). Gehen Sanktionen mit einem höheren Stigmabewusstsein bei Arbeitslosen einher? Zeitschrift für Sozialreform, 64(2), 217-248.

Henning, C. (2011). Welfare to Work: Lässt sich Arbeit sozialstaatlich erzwingen? Eine Kritik normativer Rechtfertigungen von Workfare. neue praxis, 11(Sonderheft 11), 36-46.

Hettlage, R. (2000). Masken der Kommunikation. Goffmans Hermeneutik des Selbst zwischen Nähe und Distanz. In A. Schäfer \& M. Wimmer (Hrsg.), Masken und Maskierungen (S. 161-186). Wiesbaden: Springer.

Hielscher, V., \& Ochs, P. (2009). Arbeitslose als Kunden? Beratungsgespräche in der Arbeitsvermittlung zwischen Druck und Dialog. Berlin: edition sigma.

Klammer, U., Leiber, S., \& Leitner, S. (2017). Leben im transformierten Sozialstaat: Sozialpolitische Perspektiven auf Soziale Arbeit. Soziale Passagen, 9(1), 7-21.

Kruse, J. (2014). Qualitative Interviewforschung. Ein integrativer Ansatz. Weinheim und Basel: Beltz Juventa.

Lessenich, S. (2012). „Aktivierender“ Sozialstaat. Eine politisch-soziologische Zwischenbilanz. In R. Bispinck, G. Bosch, K. Hofemann, G. Naegele \& G. Bäcker (Hrsg.), Sozialpolitik und Sozialstaat: Festschrift für Gerhard Bäcker (S. 41-53). Wiesbaden: Springer VS.

Lessenich, S., \& Mau, S. (2005). Reziprozität und Wohlfahrtsstaat. In F. Adloff \& S. Mau (Hrsg.), Vom Geben und Nehmen. Zur Soziologie der Reziprozität (S. 257-276). Frankfurt a. M.: Campus.

Lochner, B., \& Bastian, P. (2018). Sozialpädagogisches Verstehen und Beraten von asylsuchenden Menschen. Soziale Passagen, 10(2), 281-298.

Ludwig-Mayerhofer, W., Behrend, O., \& Sondermann, A. (2014). Activation, public employment services and their clients: the role of social class in a continental welfare state. Social Policy and Administration, 48(5), 594-612.

Maier-Gutheil, C. (2016). Beraten. Stuttgart: Kohlhammer.

Marquardsen, K. (2011). Eigenverantwortung ohne Selbstbestimmung? Zum Verhältnis von „Autonomie“ und Heteronomie in der aktivierenden Arbeitsmarktpolitik. Prokla, 41(2), 231-251.

Mayring, P. (2000). Qualitative Inhaltsanalyse. Forum: Qualitative Sozialforschung, 1(2), Art. 20.

McLeod, J. (2013). Introduction to counselling (5. Aufl.). Maidenhead: Open University Press.

Münder, J. (Hrsg.). (2007). Sozialgesetzbuch II. Grundsicherung für Arbeitssuchende. Lehr- und Praxiskommentar (2. Aufl.). Baden-Baden: Nomos.

Nullmeier, F. (2004). Vermarktlichung des Sozialstaats. WSI Mitteilungen, 2004(9), 495-500.

OECD (2021). Employment: time spent in paid and unpaid work by sex. https://stats.oecd.org/index.aspx? queryid=54757. Zugegriffen: 23. März 2021.

Pascall, G., \& Lewis, J. (2004). Emerging gender regimes and policies for gender equality in a wider Europe. Journal of Social Policy, 33(3), 373-394.

Rübner, M. (2018). Direktiv, reflexiv oder informativ? Beratungsstile in der Berufsberatung. neue praxis, 48(5), 487-495. 
Schäfer, M. (2019). Die Herstellung und Bewältigung von „Familiennormalität“ im Kontext des Schulbesuchs - Goffmaneske Blicke auf den Alltag von Schulkindern in familienanalogen Formen der Hilfen zur Erziehung. Soziale Passagen, 11(1), 5-25.

Schütz, H., Steinwede, J., Schröder, H., Kaltenborn, B., Wielage, N., Christe, G., \& Kupka, P. (2011). Vermittlung und Beratung in der Praxis. Eine Analyse von Dienstleistungsprozessen am Arbeitsmarkt. Bielefeld: Bertelsmann.

Senghaas, M., Bernhard, S., \& Freier, C. (2020). Eingliederungsvereinbarungen aus Sicht der Jobcenter. Pflichten der Arbeitsuchenden nehmen viel Raum ein. IAB-Kurzbericht 5/2020. Nürnberg: Institut für Arbeitsmarkt- und Berufsforschung.

Soeffner, H.-G. (2004). Auslegung des Alltags - Der Alltag der Auslegung. Frankfurt a. M.: Suhrkamp.

Sondermann, A. (2013). Zwischen subjektiven Bezügen zu Erwerbsarbeit und den Normen der Arbeitsmarktpolitik: Erwerbslose in einem schwierigen Spannungsfeld. In S. Brogi, C. Freier, U. FreierOtten \& K. Hartosch (Hrsg.), Repräsentationen von Arbeit. Transdisziplinäre Analysen und künstlerische Produktionen (S. 231-246). Bielefeld: transcript.

Sowa, F., \& Staples, R. (2013). Re-Präsentation eines idealen Arbeitsmarktes in Beratungsgesprächen der Bundesagentur für Arbeit. In S. Brogi, C. Freier, U. Freier-Otten \& K. Hartosch (Hrsg.), Repräsentationen von Arbeit. Transdisziplinäre Analysen und künstlerische Produktionen (S. 247-264). Bielefeld: transcript.

Sowa, F., \& Staples, R. (2017). Institutionelle Rahmung und Praxis des Beratens und Vermittelns. In F. Sowa \& R. Staples (Hrsg.), Beratung und Vermittlung im Wohlfahrtsstaat (S. 13-38). Baden-Baden: Nomos.

Sowa, F., \& Staples, R. (2018). Erwerbslose Arbeitssubjekte als Erwerbsarbeitssubjekte: Zur performativen Aushandlung und Festschreibung von verwalteten Biografien. In E. Schilling (Hrsg.), Verwaltete Biografien (S. 195-222). Wiesbaden: Springer VS.

Thierbach, C., \& Petschick, G. (2019). Beobachtung. In N. Baur \& J. Blasius (Hrsg.), Handbuch Methoden der empirischen Sozialforschung (2. Aufl. S. 1165-1181). Wiesbaden: Springer VS.

Thiersch, H. (2013). Soziale Arbeit in den Herausforderungen des Neoliberalismus und der Entgrenzung von Lebensverhältnissen. neue praxis, 13(3), 205-219.

Weinbach, C. (2012). Extra-vertragliche Zumutungen im New Public Cotractualism: Die doppelte Logik der Eingliederungsvereinbarung und die Rechtsstellung des Klienten im Sozialgesetzbuch II. der moderne staat, 5(2), 377-399.

Zahradnik, F., Schreyer, F., Moczall, A., Gschwind, L., \& Trappmann, M. (2016). Wenig gebildet, viel sanktioniert? Zur Selektivität von Sanktionen in der Grundsicherung des SGB II. Zeitschrift für Sozialreform, 62(2), 141-179. 